\title{
Feminisierung in der Medizin am Beispiel der Pädiatrie
}

Bea Latal,

Traudel Saurenmann ${ }^{b}$

a Leitende Ärztin, Abteilung Entwicklungspädiatrie

b Leitende Ärztin, Abteilung Rheumatologie

Keine Interessenverbindungen

\section{Korrespondenz:}

Bea Latal

Universitäts-Kinderkliniken Zürich Steinwiesstrasse 75

CH-8032 Zürich

Tel. 0442667751

Fax 0442667164

bea.latal@kispi.uzh.ch

traudel.saurenmann@kispi.uzh.ch
Die Pädiatrie ist eines der Gebiete, in denen ein sehr hoher Anteil von Frauen tätig ist. Seit Mitte der 90er Jahre werden jährlich mehr neue Facharzttitel Pädiatrie an Frauen als an Männer vergeben. Der Frauenanteil unter den berufstätigen Pädiatern betrug 2007 47\% (Abb. 1).

Wie bereits im Artikel von Jaunin et al [1] dargestellt, hat die veränderte Geschlechterverteilung weitere Folgen: Frauen arbeiten wesentlich häufiger als Männer Teilzeit, so dass mehr Ärzte ausgebildet werden müssen, damit die Versorgung der Bevölkerung sichergestellt werden kann. Frauen machen bei gleicher beruflicher Qualifikation wesentlich seltener Karriere, weder in Universität noch in Spitälern, so dass die Rekrutierung von geeignetem Nachwuchs für Kaderpositionen gefährdet ist. Der Anteil weiblicher Professoren in der Medizin liegt trotz seit Jahren ausgeglichenem Geschlechterverhältnis während der frühen ärztlichen Karriere weiterhin unter $10 \%$. Wie ausgeprägt diese «glass ceiling» an den Medizinischen Fakultäten in der Schweiz immer noch ist, wird in Abbildung 2 illustriert.

Wie können aber die Rahmenbedingungen so verändert werden, dass es für junge Ärztinnen attraktiver wird, sich für eine Spital- oder Universitätskarriere zu entscheiden und diese auch konsequent zu verfolgen? Wie können Vorgesetzte Führungsfähigkeiten bei ihren weiblichen Mitarbeitenden besser erkennen und fördern?

\section{Beispiel Kinderspital Zürich}

\section{Ausgangssituation}

Im Kinderspital Zürich gab es zur Jahrtausendwende keine habilitierten Ärztinnen, keine Leitenden Ärztinnen und keine Professorinnen. Die typische Konstellation in vielen Abteilungen war ein männlicher, habilitierter Abteilungsleiter mit einer weiblichen, nichthabilitierten Oberärztin. Typischerweise war dabei die (weibliche) Oberärztin diejenige, die sich primär um die klinische Arbeit kümmerte, während der (männliche) Abteilungsleiter für die Forschung, die Aussenkontakte (Kongresse, Publikationen, Vorträge) und das Administrative (Stellenvergabe, Zeugnisse) zuständig war.

Es erstaunt nicht, dass sich viele frischgebackene Pädiaterinnen primär ebenfalls für die

\section{La féminisation de la médecine}

Beaucoup de femmes travaillent en pédiatrie. Nous illustrons comment par des changements des conditions cadres de travail, une carrière hospitalo-universitaire peut être rendue attractive pour les femmes. Nous avons abordé cette problématique avec le projet «Optimisation des chances». Le but est de permettre un développement professionnel optimal pour les deux sexes. Le projet est divisé en sous-projets, qui permettent entre autre une meilleure qualification des médecins hommes et femmes. Un autre projet clé comprenait des séances de «coaching" pendant lesquels les médecins discutaient en groupe, sous supervision professionnelle, de leur trajectoire personnelle et de problèmes concernant leur carrière. Le projet a été réalisé avec le soutien des cadres médicaux et un accompagnement par des professionnels: en 2008 , sept femmes avaient un titre de privatdocent et neuf femmes de médecin adjoint. II reste encore des questions à aborder: la répartition encore très inégale des sexes dans les instances de décision, le travail à temps partiel comme obstacle à la carrière et la nécessité de créer des conditions de travail plus flexibles pour augmenter l'attrait des positions de cadre.

klinische Arbeit entschieden, noch besonders, da der Patientenkontakt und die psychosoziale Betreuung der Familien viele befriedigende Erlebnisse mit sich brachten. Eine Forschungskarriere war schwieriger vorstellbar und mit viel Ungewissheit verbunden, Rollenmodelle fehlten. Nur schon die Frage, was eine Habilitation genau bedeutet und welche Bedingungen erfüllt werden müssen, um in Zürich zu habilitieren, hätten die wenigsten beantworten können.

Im Sommer 2001 traf eine Gruppe von Oberärztinnen beim ärztlichen Direktor mit ihrem 
Wunsch, eine Verbesserung der beruflichen Chancen für die Ärztinnen zu diskutieren, auf offene Ohren. Mit Hilfe einer externen Beraterin wurde während der nächsten Monate das Projekt «Chancenoptimierung» entwickelt und seither umgesetzt.

Das Projekt «Chancenoptimierung» hat das Ziel, die Situation am Kinderspital so zu gestalten, dass unabhängig vom Geschlecht eine optimale berufliche Entfaltung möglich ist. Themen von verschiedenen Teilprojekten waren die Verbesse-

\section{Abbildung 1}

Frauenanteil auf verschiedenen Karrierestufen der Medizin. Anteil Frauen in Prozent auf den verschiedenen Karrierestufen von Studienbeginn bis zur Habilitation. Zahlen basierend auf Ärztestatistik FMH. Studienanfänger $=$ Anteil Frauen im 1. Studienjahr Humanmedizin, Staatsexamen = Anteil Frauen an bestandenem Staatsexamen, Dr.med $=$ Anteil Frauen an verliehenen Titeln Dr. med., Titel FMH Pädiatrie = Anteil Frauen an neu erteilten Weiterbildungstiteln FMH Pädiatrie, Habilitation Medizin = Anteil Frauen an neuen Habilitationen in der Medizin; berufstätige Ärzte = Anteil Frauen an allen berufstätigen Ärzten in der $\mathrm{CH}$; Pädiater FMH = Anteil Frauen an allen berufstätigen Pädiatern in der Schweiz.

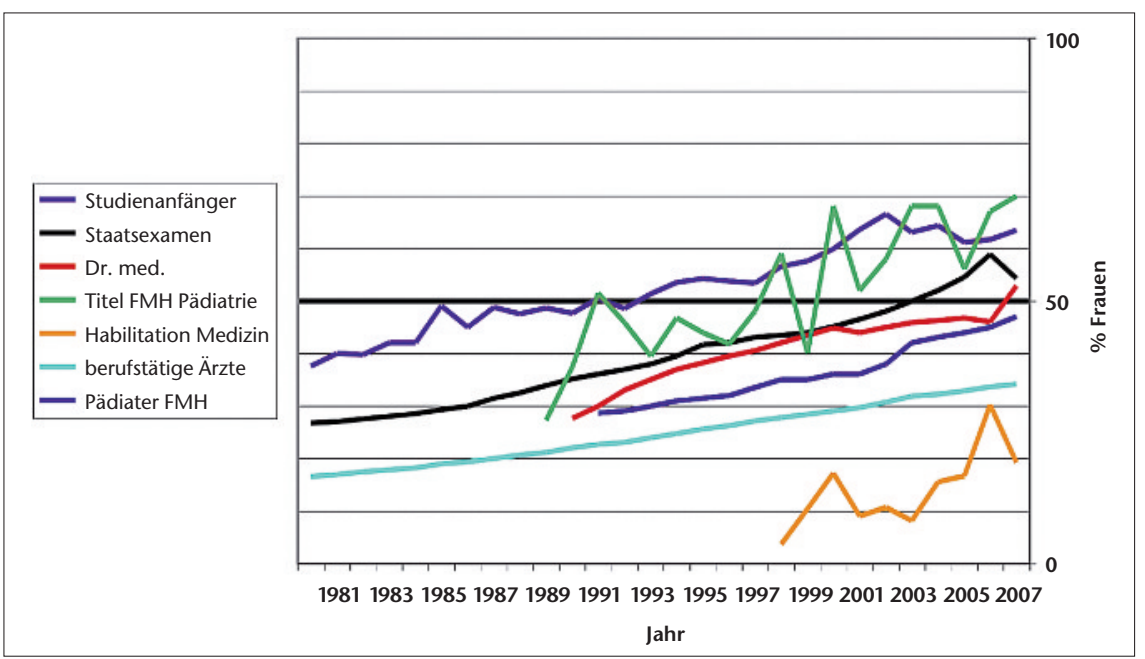

rung der Arbeitsplatzqualität, die Einführung von Qualifikationssystemen für das Kader und die Ausarbeitung flexibler Karrierewege. Als wichtige Voraussetzung für das Gelingen wurde die Verankerung innerhalb des medizinischen Kaders erkannt. Das wurde erreicht, indem in alle Teilprojekte leitende Ärzte eingebunden wurden, die sich bei der Ausarbeitung und in der Umsetzung der einzelnen Projekte engagierten. Zwei der jährlichen Kaderretraiten wurden für Teilprojekte der «Chancenoptimierung» eingesetzt und in mehreren Workshops wurde das Kader bezüglich Umsetzung der neuen Qualifikationssysteme geschult. Zentral für die beteiligten Oberärztinnen, aber auch für den Erfolg der Chancenoptimierung innerhalb des Kinderspitals, war die Aufnahme in das Bundesprogramm Chancengleichheit (siehe unten). Damit entstanden neben der Vernetzung der Ärztinnen innerhalb des Kinderspitals auch wertvolle weitere Kontakte innerhalb der Universität Zürich und zu anderen Universitäten. Höhepunkt dieser Vernetzung war die Teilnahme einer namhaften Delegation des Kinderspitals Zürich an der Tagung «Nachwuchsförderung in der Medizin - geschlechterdifferenziert» am 3. November 2004, die vom Lenkungsausschuss des Bundesprogramms Chancengleichheit der Schweizerischen Universitätskonferenz getragen wurde (www.crus.ch/ information-programme/chancengleichheit).

Im Teilprojekt «Qualifikationssysteme» wurde ein Qualifikationssystem entwickelt, das eine differenzierte Stellungnahme zu verschiedenen Aspekten der Führungsaufgabe und damit Schlussfolgerungen für die persönliche Entwicklung und Karriere ermöglicht. Insbesondere dem Thema

\section{Abbildung 2}

«Leaking Pipeline» in der Medizin. Geschlechterverteilung an den Medizinischen Fakultäten Bern und Basel. Aus der Broschüre der Tagung «Nachwuchsförderung in der Medizin geschlechterdifferenziert»

(www.cus.ch/wDeutsch/publikationen/chancengleichheit/Tagungsbericht_NWF_Medizin.pdf).

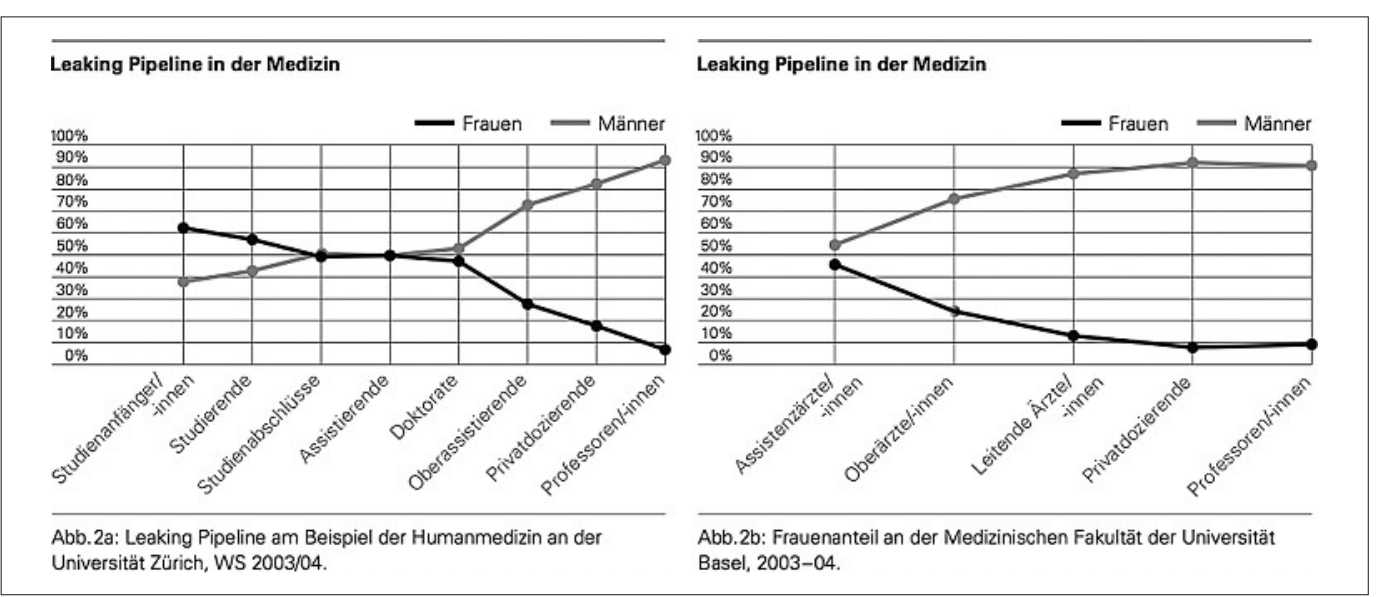


«Feedback geben» und der Beurteilung z. B. des persönlichen Verhaltens wird ein hoher Stellenwert zugeschrieben. Für Assistierende wurde ein Dossier entwickelt, mit dem sie sich, ihre Fähigkeiten und ihre Karrierewünsche selbst evaluieren können. Besonders für Ärztinnen, die oft in der Mitte oder gegen Ende ihrer Facharztausbildung vor dem Dilemma «Familie versus Karriere» stehen, ist es sehr hilfreich, sich grundsätzlich über mittel- und langfristige Karrierevorstellungen konkrete Gedanken zu machen.

Im Teilprojekt «Coaching» wird ein Gruppencoaching angeboten, bei dem 4-5 Ärztinnen mit ähnlicher Berufserfahrung unter der Leitung einer externen Fachperson berufliche Problemsituationen, persönliche Laufbahnfragen und andere Themen zur Arbeit oder Work-Life-Balance diskutieren. Pro Sitzung werden 1 bis 2 Themen besprochen, wobei die jeweils Nichtbetroffenen der Gruppe hauptsächlich in der Rolle als Zuhörer Problemlösungsstrategien erlernen. Dieses Projekt wurde in die Mentoringwerkstatt der Universität Zürich (Teil des Bundesprogramms Chancengleichheit) aufgenommen und auch aus dieser Quelle finanziert. Nach Finanzierungsende übernimmt nun das Kinderspital $2 / 3$ dieser Kosten.

Weitere Teilprojekte waren die differenzierte Darstellung und Ausarbeitung von «Karrierepfaden», die neben dem bekannten Weg ForschungHabilitation auch klinisch orientierte Laufbahnen und Laufbahnen im Bereich der Lehre oder des Managements beinhalten. Andere Themen waren der Kulturwandel innerhalb des Spitals, Teilzeitmodelle für verschiedene Arbeitsbereiche (ambulant, stationär) und deren arbeitsrechtliche Implikationen sowie geschlechterdifferenzierte Nachwuchsförderung. In diesen Arbeitsgruppen haben sich viele Ärztinnen in ihrer Freizeit über

\section{Abbildung 3}

Ärztliche Kaderstellen am Kinderspital Zürich. Entwicklung der Kaderstellen am Kinderspital Zürich (Quelle: Jahresberichte 1998-2007). Prof = Professur (Ordinariat/Extraordinariat), $\mathrm{LA}=$ Leitende/Chefärzte, $\mathrm{OA}=$ Oberärzte.

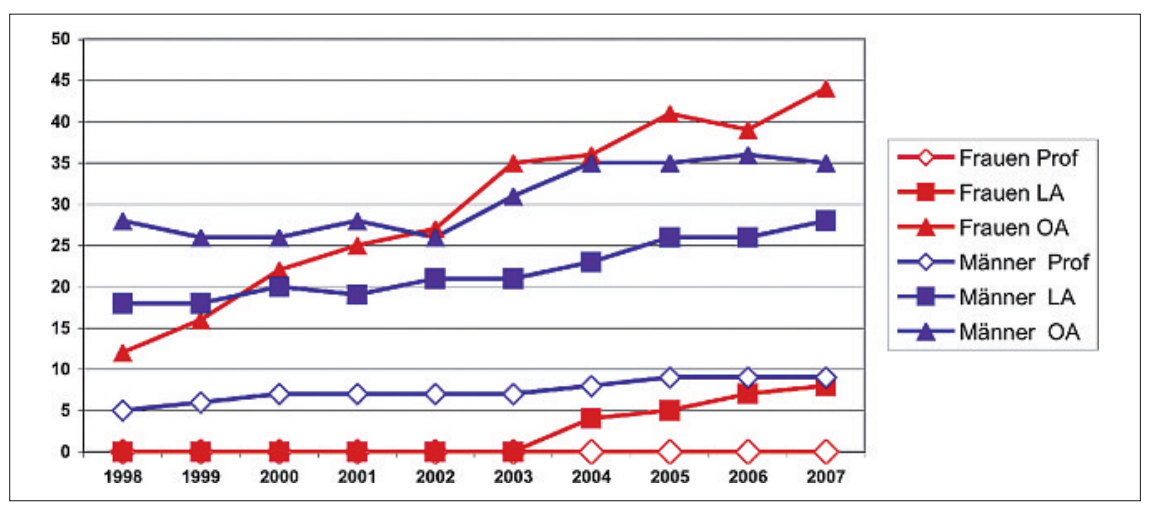

Jahre intensiv eingesetzt. Neben dem persönlichen Lerneffekt entstand so auch ein wichtiges Netzwerk für Ärztinnen innerhalb des Kinderspitals. Zudem wurden sie für die Spitalleitung im Rahmen von Besprechungen und Sitzungen «sichtbarer», was für alle einen positiven Effekt hatte.

Als konkretes Ziel wurde anlässlich der ersten Sitzung des Projekts «Chancenoptimierung» formuliert, dass bis im Jahr 2005 insgesamt 5 Ärztinnen am Kinderspital Zürich entweder habilitiert oder leitende Ärztinnen sein sollten. Dieses Ziel wurde erreicht! Im Sommer 2008 haben wir nun bereits 7 habilitierte und 9 leitende Ärztinnen (Abb. 3). Damit ist das Projekt «Chancenoptimierung» sicherlich als erfolgreich zu beurteilen.

\section{Situation am Kinderspital Zürich im Jahr 2008} Eine im März 2008 erstellte Übersicht über die Geschlechterverteilung auf den verschiedenen Hierarchiestufen und Fachbereichen (Abb. 4) überrascht in zwei Aspekten:

Trotz der obengenannten Verbesserungen ist weiterhin ein erheblicher «glass ceiling»-Effekt festzustellen: Bei den Assistenz- und Oberärzten beträgt der Frauenanteil 60\%. Dagegen ist der Frauenanteil unter den Leitenden und Chefärzten mit $20 \%$ immer noch erschreckend tief. Seit Start des Projektes Chancenoptimierung im Januar 2002 wurden am Kinderspital 18 zusätzliche Leitende/ Chefarztstellen geschaffen (je 9 Männer und Frauen) (Abb. 3). Der Effekt wirkt sich bei der grossen Gesamtzahl von Leitenden/Chefarztstellen bisher wenig aus. Natürlich spielt eine Rolle, dass die Fluktuation auf dieser Hierarchiestufe, verglichen mit Oberarzt- oder Assistierendenpositionen, naturgemäss geringer ist und der Wandel folglich erst verzögert Früchte tragen kann. Allerdings konnte bei personellem Wechsel auf Leitenden Stellen in dieser Zeit einzig die Gynäkologie wieder mit einer Frau besetzt werden, während alle anderen Nachfolger Männer waren. Es gibt am Kinderspital weiterhin keine Chefärztin oder Professorin. Fachlich letztverantwortlich sind zudem nur 4 der 9 Leitenden Ärztinnen verglichen mit 34 von 38 Leitenden/Chefärzten.

Diese Verteilung führt dazu, dass in wichtigen Arbeitsgruppen oder Gremien oft ausschliesslich Männer vertreten sind. Eine bessere Geschlechterdurchmischung in Entscheidungsgremien wäre aber nicht nur wichtig, um die Rollenvorstellungen und damit die «glass ceiling» aufzuweichen. Eine grössere Diversität führt auch nachgewiesenermassen zu fundierteren, besser abgestützten Entscheiden. Die Leitung der Universität Zürich hat hierzu einen Verhaltenskodex 


\section{Abbildung 4}

Geschlechterverteilung am Kinderspital Zürich nach Hierarchiestufe im März 2008. Der Frauen- und Männerantei im jeweiligen Bereich, umgerechnet auf Vollzeitstellen, entspricht den Angaben im System PEP (Personal-EinsatzPlanung) des Kinderspitals.

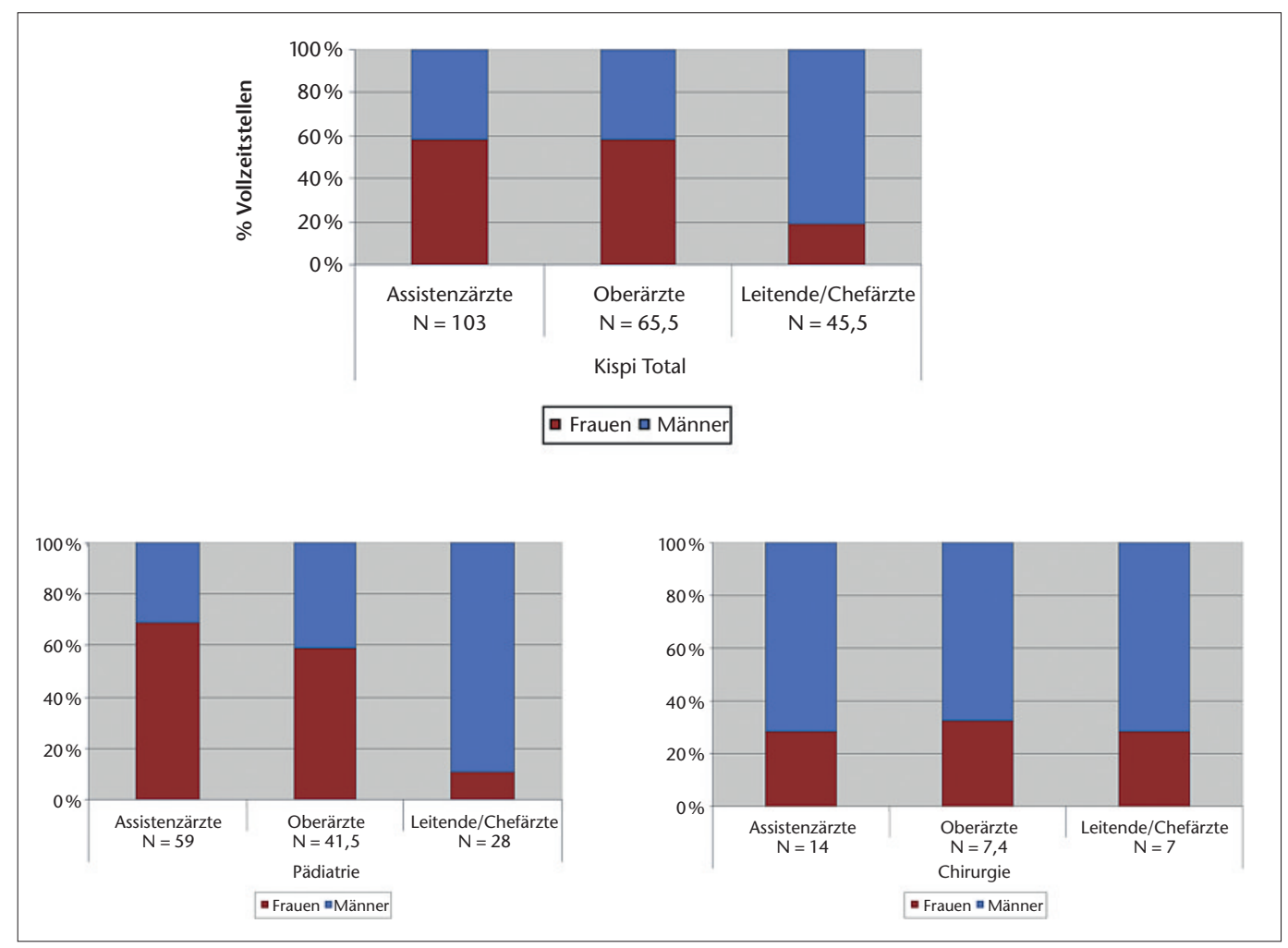

gutgeheissen, welcher auch in der Medizin umgesetzt werden sollte. (www.uzh.ch/about/basics/ genderpolicy.html)

Trennt man das Ergebnis auf nach Fachbereichen, so zeigt sich ein überraschender Unterschied zwischen Pädiatrie und Chirurgie: während der «glass ceiling»-Effekt in der Pädiatrie mit 70\% Frauen bei den Assistierenden und 10\% bei den Leitenden noch deutlicher ausfällt, ist die Geschlechterverteilung in der Chirurgie mit rund 30\% Frauenanteil auf allen Hierarchiestufen ausgeglichen.

Interessanterweise gibt es auf der Chirurgie nur sehr wenige Teilzeitstellen, darunter gar keine für Assistierende, während es in der Pädiatrie einen sehr hohen Anteil Teilzeitstellen, insbesondere auch für Assistierende, gibt - auf Wunsch der «Chancenoptimierung»! Besteht ein Zusammenhang zwischen dem Angebot von Teilzeittätigkeit und den schlechteren Karrierechancen für die Frauen in der Pädiatrie? Werden durch das Teilzeitangebot in der Pädiatrie vielleicht Mitarbeiterinnen selektioniert, die sich weniger für eine Karriere interessieren, respektive durch das fehlende Angebot in der Chirurgie solche, die besonders karrierebereit sind? Der Frage, ob das
Schaffen von Teilzeitstellen letztlich kontraproduktiv ist für die Karriereförderung von Frauen, muss unbedingt mittel- und langfristig noch einmal nachgegangen werden. Auch möglich wäre nämlich, dass es sich dabei um ein vorübergehendes Phänomen handelt und die jetzt teilzeitlich tätigen Frauen später - nach der Kleinkinderphase und eben gerade wegen der Möglichkeit, Teilzeit zu arbeiten - durchaus weitere Karriereschritte machen, siehe unten.

\section{Fazit Kinderspital Zürich}

Das Zusammentreffen einer motivierten und engagierten Gruppe von Frauen mit einer Spitalleitung, die offene Ohren für die Anliegen hat und bereit ist, sich für den Wandel in der eigenen Institution auch selbst einzusetzen, hat rasche und spürbare Ergebnisse gebracht. Gruppenzusammensetzungen und die Bereitschaft zur Zusammenarbeit sind natürlichen Schwankungen unterworfen, äussere Gegebenheiten wie Kostendruck oder allgemeine Arbeitslast können sich auf die Möglichkeiten für einen Strukturwandel auswirken. Die aktuelle Situation am Kinderspital kann und muss aber noch weiter verbessert werden. 


\section{Wo sollen weitere Verbesserungen ansetzen?}

\section{Professuren/Chefarztstellen}

Weiterhin besteht eine enorme Diskrepanz zwischen dem Anteil qualifizierter weiblicher Bewerbenden für die obersten Positionen in der Medizinhierarchie und dem Anteil schliesslich tatsächlich mit Frauen besetzter Stellen. Verschiedene Gründe können dafür genannt werden: der Mangel an Rollenmodellen, der sich sowohl bei der potentiellen Bewerberin als auch beim Wahlgremium auswirkt: Frauen kann man sich in solchen Positionen einfach weniger gut vorstellen! Dazu kommt, dass Frauen nachweislich bei gleichwertigen objektiven Qualifikationen als wissenschaftlich weniger qualifiziert beurteilt werden [2]. D.h. eine Frau muss deutlich höhere Leistungen aufweisen können - in Schweden waren es in den 90er Jahren immerhin drei Publikationen in «Nature»! -, um mit einem männlichen Mitbewerber mithalten zu können. Auch Kriterien mehr organisatorischer Natur können die Bewerbung bzw. Wahl einer Frau erschweren, z. B. der Wunsch nach einem gleichwertigen, attraktiven beruflichen Angebot für den berufstätigen Partner.

\section{Karrieremotivation von Assistenzärztinnen und Oberärztinnen}

Bei jüngeren Ärzten beiderlei Geschlechts besteht ein Trend, keine unbeschränkt hohen Arbeitszeiten mehr in Kauf zu nehmen. Teilzeitarbeit ist weit verbreitet und stösst auch zunehmend bei Männern auf Interesse. Die früher angestrebten Toppositionen (Professor, Chefarzt) verlieren an Reiz, Prestige und Attraktivität.

Wie könnte die Attraktivität von Professuren und Chefarztstellen wieder gesteigert werden? Aufgabenteilung (Lehre, Administration, Klinik, Forschung), Schaffung von Job-Sharing-Stellen, sicherere finanzielle Rahmenbedingungen und zeitliche Begrenzung der Stelle wären Möglichkeiten, wie solche Aufgaben redimensioniert werden könnten. Im Triemlispital wurde dieses Jahr erstmals in der Schweiz die Teilung einer Chefarztstelle auf der Maternité eingeführt (siehe TagesAnzeiger 11.3.08: http://sc.tagesanzeiger.ch/dyn/ news/zuerich/850026.html).

\section{Karriereplateau während der Kinderphase - Neustart danach!}

Das Dilemma Kinder-Karriere kann heutzutage besser als früher durch Teilzeitarbeit oder familienexterne Kinderbetreuung gelöst werden. Noch viel zu wenig wahrgenommen wird aber die Tatsache, dass die Phase, in der die Eltern durch die Kinderbetreuung stark absorbiert und beruflich evtl. eingeschränkt leistungsfähig sind, nach einigen Jahren vorbei ist! Im Artikel von Jaunin et al. wird von einem M-Verlauf der Karriere gesprochen [1]. Die Tatsache, dass es diese Plateauphase oder sogar einen Karriereeinbruch gibt, ist aber noch viel zu wenig anerkannt. Viele Frauen, die während Jahren Teilzeit gearbeitet haben, bekunden enorme Mühe, sich selbst und ihren Vorgesetzten klar zu machen, dass neue Karriereschritte nach dieser Zeit noch bzw. wieder möglich sind. Damit wird Teilzeitarbeit zur Karrierefalle. Diese Tatsache dürfte mit ein Grund sein, warum Teilzeittätigkeit bisher von Männern weniger oft in Betracht gezogen wird als von den im allgemeinen weniger karrierebewussten Frauen. Vorgesetzte und Betroffene müssen sich bewusst machen, dass es sich bei der Kinderbetreuung zwar um ein mehrere Jahre dauerndes, letztlich aber doch vorübergehendes Phänomen handelt. Nur wenn ganz bewusst von einem Plateau der Karriere (mit oder ohne Arbeitszeitreduktion) während der ersten intensiven Jahre der Kinderbetreuung ausgegangen wird, kann auch der Moment für einen «Neustart» prospektiv geplant werden. Damit müssen auch andere Karrieremodelle entstehen, in denen z. B. eine Habilitation nach dem Alter von 40 Jahren keinen Rechtfertigungszwang auslöst und finanzielle Unterstützung, Stipendien und Preise z. B. an die Forschungserfahrung statt an das biologische Alter gekoppelt werden.

\section{Referenzen}

1 Jaunin-Stalder N, Pécoud A, de Torrenté de la Jara G. La médicine se conjugue-t-elle au féminin? Schweiz Ärztezeitung. 2009;90(9):351-3.

2 Wennerås C, Wold A. Nepotism and sexism in peer-review. Nature. 1997;387;341-3. 